\title{
Presymptomatic and longitudinal neuroimaging in neurodegeneration-from snapshots to motion picture: a systematic review
}

\author{
Christina Schuster, Marwa Elamin, Orla Hardiman, Peter Bede
}

\begin{abstract}
- Additional material is published online only. To view please visit the journal online (http://dx.doi.org/10.1136/ jnnp-2014-309888).

Quantitative Neuroimaging Group, Academic Unit of Neurology, Biomedical Sciences Institute, Trinity College Dublin, Ireland
\end{abstract}

\section{Correspondence to} Dr Peter Bede, Room 5.43, Quantitative Neuroimaging Group, Academic Unit of Neurology, Biomedical Sciences Institute, Trinity College Dublin, Pearse Street, Dublin 2, Ireland; bedepeter@hotmail.com

Received 8 November 2014 Revised 18 December 2014 Accepted 7 January 2015

\footnotetext{
To cite: Schuster $C$, Elamin M, Hardiman O, et al. I Neurol Neurosurg Psychiatry Published Online First: [please include Day Month Year] doi:10.1136/ jnnp-2014-309888
}

\begin{abstract}
Background Recent quantitative neuroimaging studies have been successful in capturing phenotype and genotype-specific changes in dementia syndromes, amyotrophic lateral sclerosis, Parkinson's disease and other neurodegenerative conditions. However, the majority of imaging studies are cross-sectional, despite the obvious superiority of longitudinal study designs in characterising disease trajectories, response to therapy, progression rates and evaluating the presymptomatic phase of neurodegenerative conditions.

Objectives The aim of this work is to perform a systematic review of longitudinal imaging initiatives in neurodegeneration focusing on methodology, optimal statistical models, follow-up intervals, attrition rates, primary study outcomes and presymptomatic studies. Methods Longitudinal imaging studies were identified from 'PubMed' and reviewed from 1990 to 2014. The search terms 'longitudinal', 'MRI', 'presymptomatic' and 'imaging' were utilised in combination with one of the following degenerative conditions; Alzheimer's disease, amyotrophic lateral sclerosis/motor neuron disease, frontotemporal dementia, Huntington's disease, multiple sclerosis, Parkinson's disease, ataxia, HIV, alcohol abuse/ dependence.

Results A total of 423 longitudinal imaging papers and 103 genotype-based presymptomatic studies were identified and systematically reviewed. Imaging techniques, follow-up intervals and attrition rates showed significant variation depending on the primary diagnosis. Commonly used statistical models included analysis of annualised percentage change, mixed and random effect models, and non-linear cumulative models with acceleration-deceleration components.

Discussion and conclusions Although longitudinal imaging studies have the potential to provide crucial insights into the presymptomatic phase and natural trajectory of neurodegenerative processes a standardised design is required to enable meaningful data interpretation.
\end{abstract}

\section{INTRODUCTION}

In spite of their underlying pathological differences, neurodegenerative conditions share a number of strikingly common features, such as insidious onset, diagnostic uncertainty in earlyphase disease, relentless disease progression, marked clinical and genetic heterogeneity, lack of sensitive biomarkers and prognostic indicators, a long preclinical phase, and with the exception of multiple sclerosis (MS), lack of effective disease modifying agents. Moreover, many of these conditions have a relatively similar rate of decline, leading to considerable disability within a few years from diagnosis. These similarities provide the rationale to appraise research methodologies in imaging across a seemingly diverse set of conditions. While the core pathology is different in various neurodegenerative conditions, the commonly used imaging techniques, MRI pulse sequences and the methodological challenges are surprisingly similar. Furthermore, the initiatives of setting up multicentre studies, validating crossplatform protocols and establishing multicentre data repositories are also analogous, for example, the Alzheimer's disease neuroimaging initiative (ADNI), TRACK-HD or the Neuroimaging society in ALS (NISALS). While most researchers are experts of a specific neurodegenerative condition and attend disease-specific meetings, the imaging studies of other neurodegenerative conditions are an invaluable source of learning and potential methodological templates for study design.

Quantitative imaging measures have been repeatedly proposed as candidate biomarkers of neurodegenerative conditions. Non-invasive MRI-based approaches have successfully captured diseasespecific pathology in vivo in Alzheimer's disease (AD), mild cognitive impairment (MCI), frontotemporal dementia (FTD) and amyotrophic lateral sclerosis (ALS). Phenotype-specific imaging signatures have been described in variants of $\mathrm{AD}, \mathrm{FTD}^{1}$ and ALS. ${ }^{2}$ The sensitivity of specific imaging techniques has been further demonstrated by correlations between imaging parameters and clinical measures across the spectrum of neurodegenerative conditions. For example, imaging measures have been correlated with neuropsychological performance in $\mathrm{AD}^{3}{ }^{3} \mathrm{FTD}^{4}$ and with motor disability in ALS. ${ }^{2}$ Novel imaging techniques have been successfully utilised to describe genotype-specific changes, such as MAPT-related and PGRN-related changes in $\mathrm{FTD},{ }^{5}$ APO-E-associated changes in $\mathrm{AD}$ and MCI, C9orf72-specific changes in ALS, ${ }^{8}$ repeat lengthdependent changes in Huntington's disease (HD), and PRKN-related changes in in Parkinson's disease (PD). ${ }^{10}$ Imaging parameters often serve as primary end points of pharmaceutical trials in MS, but imaging-based outcome measures have also been proposed in $\mathrm{HD}^{11}$ and ALS. ${ }^{12}$ While MRI is routinely used to establish the diagnosis of MS and Ioflupane single-photon emission CT (SPECT; DaTSCAN) scan to confirm PD, recent classifier 
models also show promise of aiding the diagnosis of early ALS or $\mathrm{AD} .^{13}$ Recently, quantitative imaging techniques have been successfully utilised to highlight changes in grey and white matter integrity in presymptomatic mutation carriers in $\mathrm{HD},{ }^{14}$ $\mathrm{AD},{ }^{15} \mathrm{PD}^{16}$ and ALS. ${ }^{17}$

The majority of imaging studies in neurodegeneration are cross-sectional, even though longitudinal studies are crucial for the characterisation of natural disease trajectories, evaluation of the presymptomatic disease phase, description of genotypespecific progression rates, reporting patterns of pathological spread and appraisal of medication effects. Furthermore, longitudinal imaging has the potential to contribute to the validation of prognostic markers, establish imaging-based staging systems, define progression milestones and identify triggers for clinical interventions. Finally, multimodal longitudinal imaging studies can help to establish the relative sensitivity of the various imaging techniques.

\section{AIMS}

The aim of this study is to systematically review longitudinal neuroimaging studies in neurodegeneration, focusing on imaging methodology, sample size considerations, enrolment bias, number of time points, statistical models, follow-up intervals, attrition rates and study deliverables. An additional objective of this work is to review presymptomatic and preclinical studies in neurodegeneration and outline the optimal methodological framework for large, multicentre, longitudinal neuroimaging studies.

\section{METHODS}

Longitudinal imaging studies published between 1990 and 2014 were identified using PubMed. The search terms "longitudinal", "imaging" and "MRI" were combined with one of the following keywords: "Alzheimer's disease", "Amyotrophic lateral sclerosis", "Motor neuron disease", "Frontotemporal dementia", "Huntington disease", "Multiple sclerosis", "Parkinson's disease", "Ataxia", "HIV", "Alcohol", "Healthy ageing". A supplementary search included the term "presymptomatic" in combination with the above listed conditions. The search was performed between April and June 2014. Clinical trials, animalmodel studies and paradigm-based functional MRI studies were excluded. Studies of multiple system atrophy, progressive supranuclear palsy, Lewy body dementia, posterior cortical atrophy, and corticobasal degeneration were also excluded due to their lower incidence compared with the most common neurodegenerative conditions. While innovative longitudinal spinal cord studies have been published in $\mathrm{ALS}^{18}{ }^{19}$ and MS, ${ }^{20}$ because of the lack of longitudinal spinal cord studies in other neurodegenerative conditions, only brain imaging papers were systematically reviewed. The identified studies were individually reviewed for sample size, reference control groups, follow-up intervals, attrition rates, statistical models and their ability to capture longitudinal changes.

\section{RESULTS}

Based on the above search criteria a total of 526 publications were identified; 423 longitudinal imaging papers (online supplementary table S1) and 103 genotype-based presymptomatic studies (online supplementary table S2). One hundred and twenty-six longitudinal imaging papers were reviewed in $\mathrm{AD}$, 18 in ALS, 18 in FTD, 21 in HD, 156 in MS, 26 in PD, 3 in ataxia, 5 in HIV cognitive impairment, 7 in alcohol abuse and 43 publications in healthy ageing.
Sample size and cohort considerations

Relatively few studies disclose their formal power calculations and sample size estimations. ${ }^{21}$ The average sample size of longitudinal studies of $\mathrm{AD}$ or MS is approximately three times larger than studies of ALS or FTD (table 1). This is because of diseasespecific factors, such as sialorrhoea and orthopnoea in ALS. Behavioural impairment has implications on recruiting phenotypes of FTD cohorts, motor disability makes recruitment to ALS and MS studies difficult, and extrapyramidal impairment may limit scanning of symptomatic PD and HD. In addition to epidemiological differences in prevalence, these factors all contribute to the significant sample size differences observed in longitudinal studies across the neurodegenerative spectrum (table 1).

The reviewed articles included both prospective longitudinal projects and retrospective studies interrogating data from large international data repositories. Digital data repositories have been established for studying AD, healthy ageing, ALS and HD; such as the ADNI, NISALS ${ }^{22}$ or TRACK-HD. ${ }^{11}$ More than half of longitudinal AD studies and more than a quarter of longitudinal FTD and MS studies utilise pre-existing data banks, generating a risk of selection bias (table 1). In prospective longitudinal studies, recruitment of prevalence cases as opposed to incidence cases introduces another bias towards those who are progressing slowly, a cohort which is more likely to participate in longitudinal imaging studies.

\section{Reference groups}

It is well-established that physiological changes take place during healthy aging. ${ }^{23}$ Volume reductions and ventricular expansion has been detected at a 12-month follow-up interval. ${ }^{24}$ However, the majority of longitudinal imaging studies do not include a healthy control group, and accordingly do not account for physiological changes over time, an essential requirement to separate disease-specific effects from those associated with healthy ageing.

Additionally, very few longitudinal studies enrol neurological control cohorts. The inclusion of disease controls is particularly important in neurodegeneration, where initial symptoms may considerably overlap. Despite the advantage of characterising distinct phenotypic signatures, the longitudinal evaluation of multiple phenotypes of the same condition is seldom carried out. ${ }^{1}$

\section{Attrition rates}

Attrition rate refers to the gradual reduction of sample size over a certain period of time. Foreseeable attrition rates should ideally be estimated prior to recruitment and sample size calculations; that is, in a condition with high-attrition rates, a larger initial sample size and shorter follow-up intervals are required to draw meaningful conclusions on longitudinal changes. Few studies directly disclose their attrition rate, but most provide sufficient information for its calculation.

Attrition rates are largely condition specific, for example, due to the rapid progression of ALS the average attrition rate after 1 year is $56.2 \%$, while in MS studies it is only $5.3 \%$, in AD $6.8 \%$, in PD $19.3 \%$ and in FTD studies $28 \%$ (table 1). Interestingly, attrition rates in studies of healthy aging is higher than in MS studies indicating a possible study adherence bias of patients with chronic progressive conditions who are anxious to participate in structured monitoring programmes.

Attrition rate is also a function of the recruitment strategy; that is, carefully selected patients, who participate in clinical trials are less likely to drop out. However, participants of 
Table 1 Key characteristics of longitudinal imaging studies in neurodegeneration

\begin{tabular}{|c|c|c|c|c|c|c|c|c|c|c|}
\hline & $A D$ & ALS & FTD & HD & MS & PD & Ataxia & HIV dementia & Alcohol abuse & Healthy aging \\
\hline Reviewed studies (n) & 126 & 18 & 18 & 21 & 156 & 26 & 3 & 5 & 7 & 43 \\
\hline Sample selected from database ( $\%$ of studies) & 55.6 & 5.6 & 27.8 & 23.8 & 38.5 & 11.5 & 33.3 & 40 & 14.3 & 74.4 \\
\hline Average sample size* & 112.9 & 29.9 & 34.5 & 64.8 & 108.3 & 40.3 & 20.3 & 80 & 45.4 & 188.3 \\
\hline Median sample size* & 55 & 28 & 22 & 35 & 44 & 27.5 & 10 & 80 & 24 & 122 \\
\hline Minimum/maximum sample size* & $5 / 590$ & $11 / 48$ & $9 / 107$ & $7 / 243$ & $4 / 1465$ & $6 / 199$ & $6 / 45$ & $74 / 86$ & $15 / 111$ & $24 / 1924$ \\
\hline Average attrition rate (year 1) & 6.8 & 56.2 & 28 & 4 & 5.3 & 19.3 & $33+$ & $0+$ & $0+$ & $8+$ \\
\hline Average follow-up interval (months) $\ddagger$ & 15.7 & 5.7 & 22.2 & 15.9 & 15.4 & 29.2 & 36 & 15 & 1.7 & 34.4 \\
\hline Minimum/maximum follow-up interval & $\begin{array}{l}2 \text { weeks/ } \\
5 \text { years }\end{array}$ & $\begin{array}{l}1 \text { month/ } \\
1 \text { year }\end{array}$ & $\begin{array}{l}6 \text { months/ } \\
15 \text { years }\end{array}$ & $\begin{array}{l}6 \text { month/ } \\
2 \text { years }\end{array}$ & $\begin{array}{l}1 \text { week/ } \\
12 \text { years }\end{array}$ & $\begin{array}{l}1 \text { yearl } \\
5 \text { years }\end{array}$ & $\begin{array}{l}1 \text { yearl } \\
4.5 \text { years }\end{array}$ & $\begin{array}{l}6 \text { months/ } \\
2 \text { years }\end{array}$ & $\begin{array}{l}1 \text { week/ } \\
6 \text { months }\end{array}$ & $\begin{array}{l}2 \text { months/ } \\
12.7 \text { years }\end{array}$ \\
\hline Overall follow-up period (years) & 10 & 2.5 & 20 & 6 & 14 & 8 & 10 & 3.5 & 1 & 13 \\
\hline Average number of assessments & 2.1 & 1.6 & 2.9 & 1.4 & 4.7 & 1.6 & 1 & 1 & 1.6 & 2.7 \\
\hline Minimum/maximum number of assessments & $1 / 10$ & $1 / 4$ & $1 / 13$ & $1 / 4$ & $1 / 51$ & $1 / 3$ & $1 / 1$ & $1 / 1$ & $1 / 3$ & $1 / 10$ \\
\hline Single imaging technique (\% of studies) & 82.5 & 83.3 & 83.3 & 90.5 & 57.1 & 84.6 & 33.3 & 100 & 85.7 & 93.0 \\
\hline Multiple techniques (\% of studies) & 17.5 & 16.7 & 16.7 & 9.5 & 42.9 & 15.4 & 66.6 & 0 & 14.3 & 7.0 \\
\hline MRI studies (\% of studies) & 82.5 & 100 & 88.9 & 100 & 100 & 38.5 & 100 & 100 & 100 & 97.7 \\
\hline PET studies (\% of studies) & 19 & 0 & 16.7 & 0 & 0.6 & 50 & 0 & 0 & 0 & 7.0 \\
\hline SPECT studies (\% of studies) & 10 & 0 & 5.56 & 0 & 0 & 11.5 & 0 & 0 & 0 & 0 \\
\hline Structural MRI (\% of studies) & 78 & 38.9 & 77.8 & 95.2 & 100 & 42.3 & 100 & 80 & 85.7 & 95.35 \\
\hline Diffusion MRI (\% of studies) & 4.8 & 50 & 0 & 14.3 & 9.0 & 0 & 0 & 0 & 14.3 & 4.7 \\
\hline MR spectroscopy (\% of studies) & 0.8 & 22.2 & 0 & 0 & 5.8 & 0 & 33.3 & 20 & 14.3 & 0 \\
\hline Whole-brain analyses (\% of studies) & 34.9 & 38.9 & 27.8 & 38.1 & 55.1 & 26.9 & 66.6 & 20 & 71.4 & 34.9 \\
\hline ROI-only analyses (\% of studies) & 38.9 & 22.2 & 11.1 & 42.9 & 9.6 & 46.2 & 0 & 40 & 28.6 & 32.6 \\
\hline $\begin{array}{l}\text { Combined whole-brain and ROI analysis (\% of } \\
\text { studies) }\end{array}$ & 24.06 & 33.3 & 33.3 & 19.0 & 1.3 & 23.1 & 0 & 20 & 0 & 28.0 \\
\hline
\end{tabular}

*Values at time point 1.
tBased on a single study.

¥Attrition rate is calculated based on prospective studies only.

AD, Alzheimer's disease; ALS, amyotrophic lateral sclerosis; FTD, frontotemporal dementia; HD, Huntington's disease; MS, multiple sclerosis; PD, Parkinson's disease; PET, positron emission tomography; ROI, region of interest; SPECT, single-photon emission CT. 


\section{Neurodegeneration}

population-based incidence studies may include relatively sicker patients resulting in higher attrition rates. Ideally, the factors influencing attrition rates in longitudinal studies should be thoroughly examined and their effect on overall study results comprehensively discussed.

\section{Study methodology}

Positron emission tomography (PET)-based, SPECT-based and MRI-based techniques dominate the methodology of longitudinal studies in neurodegeneration. Very few longitudinal studies have been carried out on PET/MRI or magnetoencephalography platforms to date. Similarly, there is currently a lack of high-field, such as 7-Tesla, longitudinal MRI studies in neurodegeneration, which is likely to change dramatically in the coming years. The most commonly used grey matter MR techniques include voxelbased morphometry, cortical thickness measurements and MR spectroscopy (MRS). Frequently used white matter approaches included diffusion tensor imaging, tractography, connectomic mapping or white matter density measurements. Longitudinal imaging studies often report observations on a single white or grey matter parameter. ${ }^{19}$ Over $85 \%$ of longitudinal HD and ALS studies establish their conclusions based on a single imaging measure, most frequently an imaging proxy of atrophy. Most PD studies (84\%) also use a single imaging technique, most frequently PET. The majority of longitudinal MS and ataxia studies, however, rely on multiple, complementary methods (table 1).

A multimodal approach is clearly required to establish the relative sensitivity of the various techniques in the different disease stages. Combining multiple methods led to the observation that white matter pathology can be detected relatively early in ALS, but shows limited progression over time compared with grey matter changes, which demonstrate progressive anatomical spread in the later stages of the disease. ${ }^{25}$ Such observations on the detection thresholds of various techniques have a major impact on the design of new studies and development of viable diagnostic applications (figure 1).

In all of the neurodegenerative conditions, structural MRI is by far the most frequently utilised measure, followed by diffusion-based white matter techniques, and only a small minority of longitudinal studies use MRS for metabolic imaging. However, with the advent of whole brain MRS sequences, spectroscopy is likely to gain further momentum.

A significant proportion of $\mathrm{AD}, \mathrm{HD}$ and $\mathrm{PD}$ studies report longitudinal changes solely from a specific region of interest (ROI) such as the motor cortex or corticospinal tracts in ALS, caudate nuclei in HD, temporal lobes in AD, etc. ROI-based analyses are often performed to highlight focal changes in disease-defining brain regions; however, narrowing the ROI to a specific area introduces an anatomical selection bias and ignores the multisystem nature of these conditions. Another pitfall of 'ROI-only' studies is that considerable changes may have already taken place at the initial time point making the detection of further changes difficult. Our review suggest that less than one-third of longitudinal MRI studies report both ROI and whole-brain findings.

Currently, MRI-based techniques dominate the methodology of longitudinal imaging, despite the significant contribution of PET studies to presymptomatic and genotype-based research ${ }^{10} 26$ (table 1). With the development of novel ligands however, PET is likely to increasingly contribute to our understanding of pathophysiological changes. In spite of its cost implications, the requirement for a near-site cyclotron to produce short half-life isotopes, and limited availability, PET offers unrivalled sensitivity to identify disease-specific pathology. For example, amyloid-imaging and recently $\tau$-imaging have the potential to provide sensitive diagnostic and biomarker applications in AD. PET radioligands that bind specifically to serotonin $5 \mathrm{HT} 1 \mathrm{~A} /$ 5HT2A receptors, dopamine D2/D3 receptors, opioid mu receptors, GABA-A benzodiazepine receptors or ligands such as [11C]PMP which measures acetylcholinesterase activity enable the selective assessment of neurotransmitter-specific networks. While PET provides valuable functional insights, group-level interpretation remains challenging, and the widespread availability of MRI scanners, their high spatial resolution and relatively simple spatial registration renders the interpretation of longitudinal MR data more straightforward. This is likely to change with the availability of combined PET/MRI scanners.

\section{Statistical methods}

Most of the identified studies included 2-3 follow-up measurements with the exception of MS studies where an average of 4.7

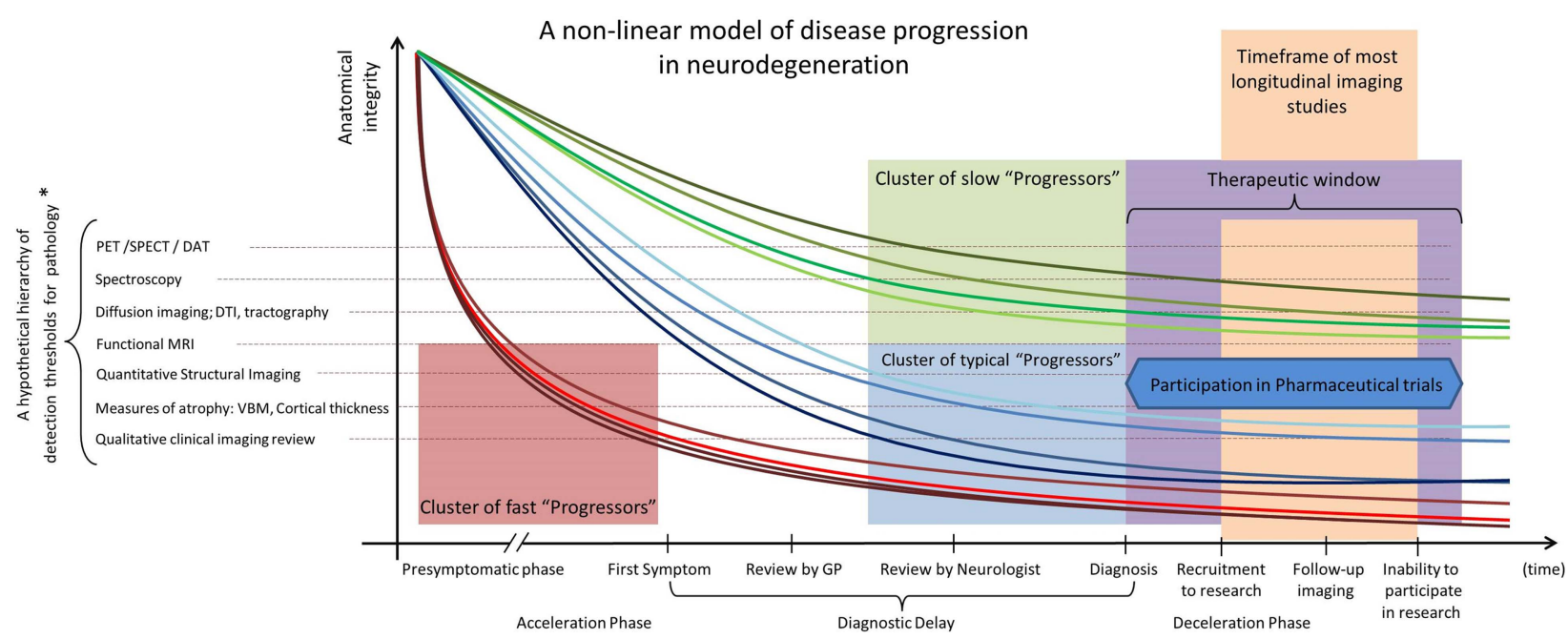

Figure 1 A schematic non-linear cumulative model of longitudinal change in neurodegeneration highlighting the effect of diagnostic delay on recruitment to pharmaceutical and longitudinal biomarker studies. The importance of multimodal, multiparametric imaging is illustrated by a hypothetical hierarchy of detection thresholds. Examples of colour coded progression rate clusters are shown. DAT, loflupane (123I) DaTSCAN; GP, general practitioner; PET, positron emission tomography; SPECT, single-photon emission CT; VBM, voxel-based morphometry; DTI, diffusion tensor imaging. 
assessments have been carried out. However, many studies have relied on a single follow-up assessment making modelling on non-linear decline impossible.

The majority of the reviewed studies have used fixed time intervals which were largely diagnosis dependent; conditions with slower progression used relatively longer follow-up periods compared with more rapid neurodegenerative conditions. The overall follow-up period from first to the last assessment also shows significant variations from a maximum of 2 years in ALS to up to 14 years in MS (table 1).

A wealth of statistical approaches was identified, ranging from relatively simple methods to complex models. Many longitudinal studies directly compare the first and second assessment, ${ }^{14} \mathrm{calcu}-$ lating the rate of change, percentage change or annualised percentage change from assuming a linear decline. ${ }^{3}$ It is well established, however, that linear models are not applicable to most neurodegenerative conditions, ${ }^{27}$ or to physiological aging ${ }^{23}$ (figure 1).

Other frequently utilised approaches include generalised estimating equations, or mixed effect linear models. The latter is particularly useful in longitudinal studies as participants with only a single assessment can be included. ${ }^{21} 2829$ This approach may also be favourable in high attrition rate studies and to model non-linear trajectories of decline. Furthermore, these models can be readily utilised to correlate longitudinal brain changes with clinical measures. ${ }^{29}$ The superiority of the mixed effects model with a random effects term, accounting for variable observation time points, compared with fixed effects analyses, has been demonstrated by a longitudinal study of $\mathrm{AD} .^{21}$ Other approaches, such as the cumulative model or sigmoid model, have been recently applied to model the rate of atrophy in $\mathrm{AD} .{ }^{30}$ This model assumes initial acceleration of volume loss up to an inflection point following which deceleration of atrophy occurs. This model has also been applied to MCI cohorts where higher acceleration rates were associated with conversion to $\mathrm{AD} .^{28}$ Acceleration-deceleration models may also explain why relatively little change is observed longitudinally in disease-defining pathological regions, such as the primary motor cortex (PMC) in ALS once significant changes have already taken place at the initial time point. ${ }^{31}$ (figure 1).

Primary regions of vulnerability, such as the PMC in ALS, the frontal lobes in behavioural variant FTD, the hippocampus in $\mathrm{AD}$, or the substantia nigra in PD are affected early and progressively (acceleration) leading to substantial pathology after which only modest further changes take place (deceleration). The 'deceleration phase' may clinically manifest as a symptomatic 'plateau' and explain why several studies fail to detect significant longitudinal changes. ${ }^{25} 32$ The same principle has been applied to neuropsychology data. The 'change point model' is based on the time at which rate of decline begins to accelerate to predict conversion to disease. ${ }^{33}$ Acceleration-deceleration models rely on the interpretation multi-time point observations to predict disease outcomes, but such models require relatively uniform interassessment intervals. Finally, multivariate classification analyses, such as the support vector machine approach, enables individual participant classifications. ${ }^{13}$

Longitudinal statistical models should ideally have the ability to manage missing data, incorporate participants with single assessments, interpret variable follow-up periods and model non-linear decline.

Cluster analysis of progression rates has implication for genetics, prognosis, diagnosis and stratification for clinical trials. ${ }^{34} 35$ Most neurodegenerative conditions, particularly ALS and PD, have a relatively uniform rate of clinical decline and short transition times between disease stages. ${ }^{36} \mathrm{~A}$ predictable disease trajectory enables the planning of support measures and the timing of assistive interventions. In patients who do not progress significantly over time or progress very slowly, the diagnosis is sometimes revisited or restrictive phenotypes are identified such as monomelic forms of ALS or primary lateral sclerosis. Conversely, studying fast 'progressors' may help to identify and characterise unique genetic or environmental factors. ${ }^{37}$ Consequently, cluster analysis of patients based on progression rates should be undertaken in studies with sufficiently large sample sizes using a combination of imaging and clinical data ${ }^{34}$ (figure 1).

\section{Presymptomatic studies}

It is widely accepted that pathological changes occur long before the diagnosis of most neurodegenerative condition. Patients often observe and report subtle changes before the diagnosis of dementia syndromes and family members often recognise behavioural changes many years before the diagnosis of FTD is formally established. It is also increasingly recognised that ALS may have long preclinical phase. ${ }^{38}$ Diagnostic delay in neurodegeneration is very significant compared with other neurological conditions and leads to considerable therapeutic delay as well as unnecessary interventions and investigations. ${ }^{39}$ As illustrated in figure 1, considerable neurodegenerative change may have taken place by the time patients are recruited into biomarker studies or pharmaceutical trials. Based on the acceleration-deceleration model, pathogenic mutation carriers in their preclinical stage are an optimal cohort to evaluate longitudinal changes. The preclinical, premanifest phase of neurodegenerative conditions offers a valuable window for pharmacological and neuroprotective intervention. Presymptomatic longitudinal neuroimaging is one of the most novel unifying research trends in neurodegeneration, an exciting interface of imaging, pathology and genetics (online supplementary table S2).

The characterisation of key presymptomatic MRI signatures in neurodegeneration assists the development of more sensitive diagnostic protocols by identifying brain regions which are affected the earliest and the techniques which are most likely to detect these early changes. Presymptomatic studies may also have prognostic ramifications, by elucidating at what point a genotype is likely to translate into symptomatic brain pathology. ${ }^{26}$ In ALS, a few pioneering studies have examined the presymptomatic phase of the disease. ${ }^{17}$ Studies of repeat length dependent syndromes, such as the hexanucleotide repeats of C9orf72, or CAG repeats in HD have revealed repeat lengthdependent structural changes. ${ }^{9}$

\section{Deliverables and outcomes of longitudinal imaging studies}

The primary objective of the reviewed longitudinal studies ranged from the characterisation of disease phenotypes ${ }^{40}$ or genotypes, ${ }^{41}$ development of prognostic indicators, ${ }^{3}$ establishment of study group-specific rates of decline, ${ }^{42}$ prediction of disease manifestation in presymptomatic cohorts, ${ }^{26}$ to the anatomical characterisation of pathological spread. ${ }^{43}$ Other proposed outcomes included the evaluation of the predictive value of baseline measures ${ }^{44}$ appraisal of the sensitivity of specific imaging techniques, ${ }^{45}$ and correlation of longitudinal imaging changes to clinical variables. ${ }^{25}$ The most common overall study conclusion was that a specific imaging measure may serve as a biomarker, which in most cases unfortunately remains aspirational. ${ }^{11}$ 


\section{Neurodegeneration}

\section{Optimal study designs}

The systematic review of longitudinal imaging in neurodegeneration enabled the identification of a core list of desirable study considerations and statistical approaches, which are relevant for the design of future prospective studies (box 1).

Despite a trend of large multicentre studies, single-site studies have clear methodological benefits. Harmonising pulsesequences and analysis pipelines across different field-strengths, manufacturer platforms and raw data formats requires complex quality control and validation procedures with phantom studies. Such studies are routinely undertaken in pharmacological studies of MS, but have to be carefully justified in a research setting. Even longitudinal single-centre, single-scanner studies are occasionally compromised by software updates, novel head

Box 1 Desirable design features of longitudinal studies

in neurodegeneration

\section{Study designs}

- Performance of whole-brain and region of interest (ROI) analyses

- Evaluation of multiple grey and white matter imaging parameters (structural, diffusion, metabolic) in the same study or multiplatform positron emission tomography/MRI measures

- Incidence-based studies

- Comprehensive clinical phenotyping and genotyping

- Multiple (more than two time point) designs

- Inclusion of presymptomatic/premanifest cohorts

- Inclusion of a longitudinal healthy control group

- Inclusion of disease and neurological 'mimic' control group

- Collection of longitudinal clinical data after imaging is no longer possible for validation of prognostic markers

- Incorporation of standardised imaging protocols into pharmaceutical trials

- Postmortem brain banking where available

Desirable statistical frameworks

- Interpretation of variable follow-up periods

- Ability to model non-linear decline

- Correlations with clinical variables

- Cluster analysis of progression rates

- Multivariate classification analyses (support vector machine approach) to classify individual patients

- Comparative evaluation of the sensitivity of various imaging techniques

- Development, evaluation and validation of prognostic markers based on initial time point or multiple time point imaging data.

- Definition of stage defining imaging measures

\section{Disclosures and discussions}

- Formal power and sample size calculations for established modalities

- Attrition rate estimations prior to recruitment

- Disclosure of recruitment strategies; incidence or prevalence cases

- Presentation of attrition rates, discussing factors contributing to attrition rates and discussion of the impact of attrition on overall findings

- Disclosure of negative results, that is, lack of progression in specific ROls coils, modification of pulse sequences which requires stringent postprocessing corrections.

\section{DISCUSSION}

In this review, we have captured the academic, clinical and pharmaceutical potential of high-quality longitudinal studies in neurodegeneration (box 2). It is clear that longitudinal neuroimaging offers superior descriptive power to cross-sectional studies and is undoubtedly the optimal method to study progressive conditions. One of the biggest challenges of longitudinal analyses is the non-linear nature of disease progression, coupled with the relatively late recruitment of patients to biomarker studies (figure 1). Presymptomatic studies, early recruitment to biomarker studies at the time of diagnosis, initiation of incidence-based prospective studies, multicentre collaborations, comprehensive genotyping and implementation of robust statistical models are just some of the options that may enhance longitudinal imaging studies. The expected attrition rate associated with the target disease should be taken into account prior to study onset for accurate power calculations. This has cost implications for conditions with high attrition rates. Longitudinal scanning using MRI should seek to combine multiple complementary imaging modalities for sensitivity analyses.

Cross-sectional studies often attempt to match patient groups for disease duration from symptom onset to the date of scan ${ }^{46}$ assuming relatively uniform progression rates. Given the differences in rate of decline however, this could be viewed as a suboptimal approach. Longitudinal imaging can address this concern by generating models where single time point and longitudinal multipoint data can be integrated. Single time point imaging data can also be utilised to validate prognostic models

Box 2 Potential deliverables of longitudinal studies in neurodegeneration

Pathophysiological insights

- Characterisation of genotype-specific rate of decline, anatomical spread

- Characterisation of phenotype-specific rate of decline, anatomical spread

- Identification of distinct disease stages based on objective criteria

- Development and validation of accurate prognostic indicators

- Description of premanifest, presymptomatic pathological changes

- Identification of an optimal therapeutic window

Clinical applications

- Diagnostic applications: patient classification based on longitudinal changes

- Prognostic indicators; introduction and withdrawal of interventions

- Assessment for rehabilitation potential

Pharmaceutical applications

- Quantification of disease burden prior to therapy

- Patient stratification based on predicted disease trajectories; fast versus slow 'progressors'

- Evaluation of response to therapy

- Discrimination of symptomatic versus disease modifying effect 
by subsequent clinical assessments, when imaging may no longer be possible. Cluster analyses of longitudinal data using clinical, imaging and genetic data have the potential to identify protective factors in 'slow progressors' and potential risk factors in 'fast progressors'. The identification of 'atypical' patients based on objective imaging data makes targeted deep-phenotyping possible and may help to explore unique environmental and genetic factors in these cohorts.

One of the academic deliverables of well-designed longitudinal studies is the confirmation or rejection of attractive, but empirical theories, such as 'focal spreading', 'spread along functional connections', 43 'dying-back', 'dying-forward', 'wires together-dies together' ${ }^{47}$ hypotheses. The time interval from first symptom to definite diagnosis is often regarded as a prognostic indicator, suggesting that the longer it takes to establish the diagnosis the slower the progression rate may be. ${ }^{48}$ Using well-constructed and adequately powered longitudinal imaging protocols, the current technology is now well placed to objectively examine these clinical observations.

Disease-state 'staging' is a fundamental medical concept widely used in neurology, oncology and other specialties. It enables patient stratification into clinical trials and examining the evidence for the introduction and withdrawal of treatment in progressive conditions. Clinical staging frameworks are extensively used in neurodegeneration, for example, Hoehn and Yahr in PD, El Escorial in ALS. Pathological staging systems, such as the Braak staging in $\mathrm{AD}$, or the recently proposed TDP-43-based staging in ALS, are used for postmortem characterisation in neurodegeneration. Novel initiatives in biomarker research have the capability to integrate the two approaches by developing objective, imagingbased staging systems that rely on measurement of disease-specific pathological change in vivo. ${ }^{49}$ If 'staging' represents artificially defined phases of progressive conditions, functional 'rating scales' reflect on the continuum of clinical changes. Unified Parkinson Disease Rating Scale (UPDRS), Expanded Disability Status Scale (EDSS), Revised ALS functional rating scale (ALSFRS-r), Alzheimer's Disease Assessment Scale-Cognitive (ADAS-Cog), International Cooperative Ataxia Rating Scale (ICARS) are just some of the disease-specific clinical rating scales which are frequently utilised as end points of clinical trials. As has been demonstrated in MS, quantitative imaging protocols have the potential to complement these scales in neurodegeneration, and to assess response to therapy in randomised controlled trials by objectively measuring longitudinal changes in the various study groups. ${ }^{50}$

\section{CONCLUSIONS}

Longitudinal studies in neurodegeneration are superior to crosssectional studies in characterising specific disease phenotypes and genotypes. Well-designed longitudinal studies have both academic merits and important clinical benefits. They enable the characterisation of genotype-specific progression curves and presymptomatic changes, as well as the development of prognostic markers, staging of the disease and accurate measurement of response to therapy. While cross-sectional studies provide useful snapshots of group level pathology, longitudinal neuroimaging studies have the potential to dissect the dynamic and active biological processes underpinning neurodegeneration. The methodological challenges of longitudinal imaging are similar across the spectrum of neurodegenerative conditions, such as non-linear disease progression curves, high attrition rates, heterogeneous patient cohorts and relatively late recruitment to biomarker studies. Presymptomatic and prospective incidence-based studies utilising robust statistical models to interpret multimodal imaging data are likely to overcome these methodological challenges.
Funding This work was supported by the Elan Fellowship in Neurodegeneration, the Health Research Board and the Research Motor Neuron (RMN-Ireland) foundation. $\mathrm{OH}^{\prime} \mathrm{s}$ research group has also received funding from the European Community's Seventh Framework Programme (FP7/2007-2013) under grant agreement $\mathrm{n}^{\circ}$ [259867] (EUROMOTOR), the EU-Joint Programme for Neurodegeneration (JPND) SOPHIA project and an unrestricted research grant from Elan Pharmaceuticals.

Competing interests $\mathrm{OH}$ has received speaking honoraria from Janssen Cilag, Biogen Idec, Sanofi Aventis and Merck-Serono, she has been a member of advisory panels for Biogen Idec, Allergen, Cytokinetics, Ono Pharmaceuticals and Sanofi.

Provenance and peer review Not commissioned; externally peer reviewed.

\section{REFERENCES}

1 Lam BY, Halliday GM, Irish M, et al. Longitudinal white matter changes in frontotemporal dementia subtypes. Hum Brain Mapp 2014;35:3547-57.

2 Bede P, Bokde A, Elamin M, et al. Grey matter correlates of clinical variables in amyotrophic lateral sclerosis (ALS): a neuroimaging study of ALS motor phenotype heterogeneity and cortical focality. J Neurol 2013;84:766-73.

3 Grothe $M$, Heinsen $H$, Teipel S. Longitudinal measures of cholinergic forebrain atrophy in the transition from healthy aging to Alzheimer's disease. Neurobiol Aging 2013:34:1210-20.

4 Knopman DS, Jack CR Jr, Kramer JH, et al. Brain and ventricular volumetric changes in frontotemporal lobar degeneration over 1 year. Neurology 2009;72:1843-9.

5 Whitwell JL, Jack CR Jr, Boeve BF, et al. Voxel-based morphometry patterns of atrophy in FTLD with mutations in MAPT or PGRN. Neurology 2009;72:813-20.

6 Lu PH, Thompson PM, Leow A, et al. Apolipoprotein E genotype is associated with temporal and hippocampal atrophy rates in healthy elderly adults: a tensor-based morphometry study. J Alzheimers Dis 2011;23:433-42.

7 Bede P, Bokde AL, Byrne $S$, et al. Multiparametric MRI study of ALS stratified for the C9orf72 genotype. Neurology 2013;81:361-9.

8 Bede $\mathrm{P}$, Elamin $\mathrm{M}$, Byrne $\mathrm{S}$, et al. Basal ganglia involvement in amyotrophic lateral sclerosis. Neurology 2013;81:2107-15.

9 Ruocco HH, Bonilha L, Li LM, et al. Longitudinal analysis of regional grey matter loss in Huntington disease: effects of the length of the expanded CAG repeat. J Neurol Neurosurg Psychiatry 2008;79:130-5.

10 Pavese N, Moore RY, Scherfler C, et al. In vivo assessment of brain monoamine systems in parkin gene carriers: a PET study. Exp Neurol 2010;222:120-4.

11 Tabrizi SJ, Reilmann R, Roos RA, et al. Potential endpoints for clinical trials in premanifest and early Huntington's disease in the TRACK-HD study: analysis of 24 month observational data. Lancet Neurol 2012;11:42-53.

12 Kalra S, Tai $P$, Genge A, et al. Rapid improvement in cortical neuronal integrity in amyotrophic lateral sclerosis detected by proton magnetic resonance spectroscopic imaging. J Neurol 2006;253:1060-3.

13 Orru G, Pettersson-Yeo W, Marquand AF, et al. Using Support Vector Machine to identify imaging biomarkers of neurological and psychiatric disease: a critical review. Neurosci Biobehav Rev 2012;36:1140-52.

14 Aylward EH, Sparks BF, Field KM, et al. Onset and rate of striatal atrophy in preclinical Huntington disease. Neurology 2004;63:66-72.

15 Fox NC, Warrington EK, Freeborough PA, et al. Presymptomatic hippocampal atrophy in Alzheimer's disease. A longitudinal MRI study. Brain 1996;119 (Pt 6): 2001-7.

16 Tang CC, Poston KL, Dhawan V, et al. Abnormalities in metabolic network activity precede the onset of motor symptoms in Parkinson's disease. J Neurosci 2010;30:1049-56.

17 Turner MR, Hammers A, Al-Chalabi A, et al. Distinct cerebral lesions in sporadic and 'D90A' SOD1 ALS: studies with [11C]flumazenil PET. Brain 2005;128(Pt 6): 1323-9.

18 Agosta F, Rocca MA, Valsasina P, et al. A longitudinal diffusion tensor MRI study of the cervical cord and brain in amyotrophic lateral sclerosis patients. I Neurol Neurosurg Psychiatry 2009;80:53-5.

19 El Mendili MM, Cohen-Adad J, Pelegrini-Issac M, et al. Multi-parametric spinal cord MRI as potential progression marker in amyotrophic lateral sclerosis. PLOS ONE 2014:9:e95516.

20 Freund $\mathrm{P}$, Wheeler-Kingshott $\mathrm{C}$, Jackson J, et al. Recovery after spinal cord relapse in multiple sclerosis is predicted by radial diffusivity. Mult Scler 2010;16:1193-202.

21 Teipel SJ, Peters 0, Heuser I, et al. Atrophy outcomes in multicentre clinical trials on Alzheimer's disease: effect of different processing and analysis approaches on sample sizes. World J Biol Psychiatry 2011;12(Suppl 1):109-13.

22 Turner MR, Grosskreutz J, Kassubek J, et al. Towards a neuroimaging biomarker for amyotrophic lateral sclerosis. Lancet Neurol 2011;10:400-3.

23 Fjell AM, Westlye LT, Grydeland $\mathrm{H}$, et al. Critical ages in the life course of the adult brain: nonlinear subcortical aging. Neurobiol Aging 2013;34:2239-47.

24 Fjell AM, McEvoy L, Holland D, et al. Brain changes in older adults at very low risk for Alzheimer's disease. J Neurosci 2013;33:8237-42. 
25 Menke RA, Korner S, Filippini N, et al. Widespread grey matter pathology dominates the longitudinal cerebral MRI and clinical landscape of amyotrophic lateral sclerosis. Brain 2014;137(Pt 9):2546-55.

26 Ciarmiello A, Giovacchini G, Orobello S, et al. 18F-FDG PET uptake in the pre-Huntington disease caudate affects the time-to-onset independently of CAG expansion size. Eur J Nucl Med Mol Imaging 2012;39:1030-6.

27 McDonald CR, McEvoy LK, Gharapetian L, et al. Regional rates of neocortical atrophy from normal aging to early Alzheimer disease. Neurology 2009; 73:457-65.

28 Leung KK, Bartlett JW, Barnes J, et al. Cerebral atrophy in mild cognitive impairment and Alzheimer disease: rates and acceleration. Neurology 2013:80:648-54

29 Nowrangi MA, Lyketsos CG, Leoutsakos JM, et al. Longitudinal, region-specific course of diffusion tensor imaging measures in mild cognitive impairment and Alzheimer's disease. Alzheimers Dement 2013;9:519-28.

30 Sabuncu MR, Desikan RS, Sepulcre J, et al. The dynamics of cortical and hippocampal atrophy in Alzheimer disease. Arch Neurol 2011;68:1040-8.

31 Verstraete $\mathrm{E}$, Veldink JH, van den Berg $\mathrm{LH}$, et al. Structural brain network imaging shows expanding disconnection of the motor system in amyotrophic lateral sclerosis. Hum Brain Mapp 2014;35:1351-61.

32 Laakso MP, Lehtovirta M, Partanen K, et al. Hippocampus in Alzheimer's disease: a 3-year follow-up MRI study. Biol Psychiatry 2000;47:557-61.

33 Hall $C B$, Lipton RB, Sliwinski $M$, et al. A change point model for estimating the onset of cognitive decline in preclinical Alzheimer's disease. Stat Med 2000;19:1555-66.

34 Gomeni R, Fava M. Amyotrophic lateral sclerosis disease progression model. Amyotroph Lateral Scler frontotemporal degener 2014;15:119-29.

35 Simon NG, Turner MR, Vucic $S$, et al. Quantifying disease progression in amyotrophic lateral sclerosis. Ann Neurol 2014;76:643-57.

36 Balendra $\mathrm{R}$, Jones $\mathrm{A}$, Jivraj $\mathrm{N}$, et al. Use of clinical staging in amyotrophic lateral sclerosis for phase 3 clinical trials. J Neurol Neurosurg Psychiatry 2015;86:45-9.

37 Byrne S, Elamin M, Bede P, et al. Cognitive and clinical characteristics of patients with amyotrophic lateral sclerosis carrying a C9orf72 repeat expansion: a population-based cohort study. Lancet Neurol 2012;11:232-40.
38 Eisen A, Kiernan M, Mitsumoto $\mathrm{H}$, et al. Amyotrophic lateral sclerosis: a long preclinical period? J Neurol Neurosurg Psychiatry 2014;85:1232-8.

39 Cellura E, Spataro R, Taiello AC, et al. Factors affecting the diagnostic delay in amyotrophic lateral sclerosis. Clin Neurol Neurosurg 2012;114:550-4.

40 van der Graaff MM, Sage CA, Caan MW, et al. Upper and extra-motoneuron involvement in early motoneuron disease: a diffusion tensor imaging study. Brain 2011;134(Pt 4):1211-28.

41 Rohrer JD, Ridgway GR, Modat M, et al. Distinct profiles of brain atrophy in frontotemporal lobar degeneration caused by progranulin and tau mutations. Neuroimage 2010;53:1070-6.

42 Frings L, Mader I, Landwehrmeyer BG, et al. Quantifying change in individual subjects affected by frontotemporal lobar degeneration using automated longitudinal MRI volumetry. Hum Brain Mapp 2012;33:1526-35.

43 Verstraete $\mathrm{E}$, van den Heuvel MP, Veldink JH, et al. Motor network degeneration in amyotrophic lateral sclerosis: a structural and functional connectivity study. PLOS ONE 2010;5:e13664.

44 Tabrizi SJ, Scahill RI, Owen G, et al. Predictors of phenotypic progression and disease onset in premanifest and early-stage Huntington's disease in the TRACK-HD study: analysis of 36-month observational data. Lancet Neurol 2013;12:637-49.

45 Acosta-Cabronero J, Alley S, Williams GB, et al. Diffusion tensor metrics as biomarkers in Alzheimer's disease. PLOS ONE 2012;7:e49072.

46 Bede $\mathrm{P}$, Elamin $\mathrm{M}$, Byrne $\mathrm{S}$, et al. Patterns of cerebral and cerebellar white matter degeneration in ALS. J Neurol Neurosurg Psychiatry Published Online First: 21 July 2014. doi:10.1136/jnnp-2014-308172

47 Bak TH, Chandran S. What wires together dies together: verbs, actions and neurodegeneration in motor neuron disease. Cortex 2012;48:936-44.

48 Chio A, Logroscino G, Hardiman 0, et al. Prognostic factors in ALS: a critical review. Amyotroph Lateral Scler 2009;10:310-23.

49 Kassubek J, Muller HP, Del Tredici K, et al. Diffusion tensor imaging analysis of sequential spreading of disease in amyotrophic lateral sclerosis confirms patterns of TDP-43 pathology. Brain 2014;137(Pt 6):1733-40.

50 Radue EW, O'Connor P, Polman $\mathrm{CH}$, et al. Impact of fingolimod therapy on magnetic resonance imaging outcomes in patients with multiple sclerosis. Arch Neurol 2012;69:1259-69. 


\section{JNNP}

Presymptomatic and longitudinal neuroimaging in neurodegeneration--from snapshots to motion picture: a systematic review

Christina Schuster, Marwa Elamin, Orla Hardiman and Peter Bede

J Neurol Neurosurg Psychiatry published online January 28, 2015

Updated information and services can be found at:

http://jnnp.bmj.com/content/early/2015/01/28/jnnp-2014-309888

These include:

Supplementary Supplementary material can be found at: Material http://jnnp.bmj.com/content/suppl/2015/01/28/jnnp-2014-309888.DC1.

References This article cites 49 articles, 12 of which you can access for free at: http://jnnp.bmj.com/content/early/2015/01/28/jnnp-2014-309888\#BIBL

Email alerting Receive free email alerts when new articles cite this article. Sign up in the service box at the top right corner of the online article.

Topic Articles on similar topics can be found in the following collections Collections

Drugs: CNS (not psychiatric) (1789)

Dementia (944)

Memory disorders (psychiatry) (1291)

Parkinson's disease (626)

HIV/AIDS (97)

Immunology (including allergy) (1745)

Motor neurone disease (275)

Movement disorders (other than Parkinsons) (756)

Multiple sclerosis (835)

Neuromuscular disease (1222)

Psychiatry of old age (317)

Spinal cord (491)

\section{Notes}

To request permissions go to:

http://group.bmj.com/group/rights-licensing/permissions

To order reprints go to:

http://journals.bmj.com/cgi/reprintform

To subscribe to BMJ go to:

http://group.bmj.com/subscribe/ 